\title{
A PULVERIZAÇÃO PRÉ-COLHEITA COM ÁCIDO GIBERÉLICO (GA $)$ E AMINOETOXIVINILGLICINA (AVG) RETARDA A MATURAÇÃO E REDUZ AS PERDAS DE FRUTOS NA CULTURA DO PESSEGUEIRO ${ }^{1}$
}

\author{
CASSANDRO VIDAL TALAMINI DO AMARANTE², AMANDA MARIA FURTADO DREHMER ${ }^{3}$, \\ FRANCIELLE DE SOUZA ${ }^{3}$, POLIANA FRANCESCATTO ${ }^{3}$
}

\begin{abstract}
RESUMO - Este trabalho objetivou avaliar os efeitos da pulverização pré-colheita com ácido giberélico (GA (G $_{3}$ e aminoetoxivinilglicina (AVG) na queda pré-colheita, maturação e qualidade de pêssegos, da cultivar Rubidoux. O delineamento experimental foi em blocos inteiramente casualizados, com quatro repetições, consistindo de seis tratamentos, resultantes da combinação de duas doses de GA 3 ( 0 e 100 mg L ${ }^{-1}$ ) e três doses de AVG (0; 75 e 150 $\left.\mathrm{mg} \mathrm{L}^{-1}\right)$. O GA 3 e o AVG foram pulverizados cerca de seis e três semanas antes do início da colheita comercial dos frutos, respectivamente. Tratamentos envolvendo a combinação de $\mathrm{GA}_{3}\left(100 \mathrm{mg} \mathrm{L}^{-1}\right)$ e AVG $\left(75\right.$ e $\left.150 \mathrm{mg} \mathrm{L}^{-1}\right)$ retardaram a maturação dos frutos na colheita e durante o armazenamento refrigerado (4 semanas a $0-2^{\circ} \mathrm{C} / 90-95 \%$ UR), ocasionando maior retenção de cor verde da casca, menor redução da firmeza de polpa, menor aumento no teor de sólidos solúveis totais e menor redução na acidez titulável. De forma geral, os efeitos mais expressivos foram observados para tratamentos com GA $100 \mathrm{mg} \mathrm{L}^{-1}$ do que para tratamentos com AVG 75 e $150 \mathrm{mg} \mathrm{L}^{-1}$. O tratamento com GA $100 \mathrm{mg} \mathrm{L}^{-1}$ também reduziu o número de frutos com rachaduras e podridões, aumentou o peso médio de frutos na colheita e reduziu a incidência de escurecimento da polpa após armazenamento refrigerado. O AVG aumentou a incidência de frutos rachados.
\end{abstract}

Termos para indexação: Prunus persica L., giberelina, etileno, queda pré-colheita, doenças, distúrbios fisiológicos, qualidade pós-colheita.

\section{PREHARVEST SPRAYING WITH GIBBERELLIC ACID (GA) AND AMINOETHOXYVINILGLYCINE (AVG) DELAYS FRUIT MATURITY AND REDUCES FRUIT LOSSES ON PEACHES}

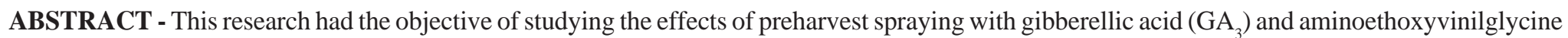
(AVG) on preharvest fruit drop, fruit maturity and fruit quality of peach, cultivar Rubidoux. The experiment consisted of a randomized complete block design, with four replicates, comprising six treatments derived from combinations of two doses of $\mathrm{GA}_{3}\left(0\right.$ and $\left.100 \mathrm{mg} \mathrm{L}^{-1}\right)$ and three doses of AVG $(0$, 75, and $\left.150 \mathrm{mg} \mathrm{L}^{-1}\right)$. GA $\mathrm{G}_{3}$ and AVG were sprayed about six and three weeks before commercial fruit harvest, respectively. Treatments with GA $\mathrm{H}_{3}(100 \mathrm{mg}$ $\mathrm{L}^{-1}$ ) and AVG ( 75 and $150 \mathrm{mg} \mathrm{L}^{-1}$ ) delayed fruit maturity at harvest and during cold storage ( 4 weeks at $0-2^{\circ} \mathrm{C} / 90-95 \% \mathrm{RH}$ ), resulting in a better retention of skin background color, the least reduction of flesh firmness, the least increasing of acidity, and the least increasing of soluble solids content ( $\left.{ }^{\circ} \mathrm{Brix}\right)$. The treatment with $\mathrm{GA}_{3}\left(100 \mathrm{mg} \mathrm{L}^{-1}\right)$ was more effective than AVG (75 and $\left.150 \mathrm{mg} \mathrm{L}^{-1}\right)$ to delay fruit maturity. In addition, GA 3 reduced the number of fruit with skin splitting and decay, increased the fruit average weight at harvest, and reduced the incidence of flesh browning after cold storage. The AVG increased fruit skin splitting.

Index terms: Prunus persica L., gibberellin, ethylene, fruit preharvest drop, decay, disorders, postharvest quality.

\section{INTRODUÇÃO}

A cultura do pessegueiro tem apresentado substancial expansão na área plantada no Sul do Brasil, representando uma importante fonte de renda para os produtores rurais. Santa Catarina é o segundo maior produtor de pêssegos, com uma área cultivada de 4.187 ha no ano de 2000, sendo apenas superado pelo Rio Grande do Sul, com uma área de 10.516 ha (Matos, 2000).

A oferta de pêssego no mercado Catarinense ocorre em curto período de tempo, resultante do pequeno intervalo de colheita entre cultivares precoces e tardias plantadas e do reduzido potencial de conservação da qualidade do produto em refrigeração. O pêssego apresenta elevada predisposição a distúrbios fisiológicos pós-colheita, relacionados à senescência, levando à ocorrência de lanosidade e degenerescência e escurecimento interno da polpa, ocasionando elevadas perdas de qualidade e reduzida longevidade de armazenamento (Wills et al., 1998).

O pêssego é um fruto climatérico, apresentando um aumento respiratório e de produção de etileno na fase de maturação (MartínezRomero et al., 2000). O aumento na produção de etileno ocasiona alterações fisiológicas, físicas e químicas relacionadas à maturação e senescência dos frutos (Taiz \& Zeiger, 1998; Wills et al., 1998). Portanto, a preservação da qualidade pós-colheita em pêssegos pode ser obtida pela redução da síntese e/ou ação do etileno (Wills et al., 1998).

Fitorreguladores podem ser utilizados para retardar a maturação e preservar a qualidade pós-colheita de frutos. O tratamento pré-colheita ou pós-colheita com ácido giberélico $\left(\mathrm{GA}_{3}\right)$ retarda a maturação em pêssego
(Ju et al., 1999; Martínez-Romero et al., 2000). O composto aminoetoxivinilglicina (AVG) é um potente inibidor da biossíntese de etileno, impedindo a conversão de S-adenosil-metionina em ácido 1-carboxi-1amino-ciclopropano (ACC) (Taiz \& Zeiger, 1998). Em maçãs, a aplicação pré-colheita deste produto retardou o aumento na produção de etileno, retardando a maturação e reduzindo a queda pré-colheita e o amadurecimento dos frutos durante o armazenamento refrigerado (Amarante et al., 2002; Autio \& Bramlage, 1982). A pulverização pré-colheita de pessegueiro com AVG (500 $\left.\mathrm{mg} \mathrm{L}^{-1}\right)$, bem como a imersão em pós-colheita de pêssegos (em doses de até $333 \mathrm{mg} \mathrm{L}^{-1}$ ) e nectarinas (em doses de até $2.664 \mathrm{mg} \mathrm{L}^{-1}$ ) retardou a maturação dos frutos (Byers, 1997). Ju et al. (1999) observaram que a pulverização de pessegueiros com AVG (em doses de até $150 \mathrm{mg} \mathrm{L}^{-1}$ ), duas semanas antes do início da colheita comercial, apresentou efeito significativo no retardo da maturação e do amadurecimento e na redução da incidência de escurecimento interno da polpa nos frutos. Os mesmos autores também observaram que a pulverização com $\mathrm{GA}_{3}$ (100 e $150 \mathrm{mg} \mathrm{L}^{-1}$ ), no estádio correspondente ao final do endurecimento do endocarpo, também apresentou este efeito, ocorrendo resposta sinergística no fruto quando da aplicação de $\mathrm{GA}_{3} \mathrm{em}$ combinação com AVG. O retardo na maturação dos frutos nos tratamentos com AVG e GA resultou em aumento no peso médio dos frutos na colheita. Estes resultados mostram que tratamentos à base de AVG, em combinação com $\mathrm{GA}_{3}$, representam grande potencial para utilização em pomares comerciais de pêssego, com a finalidade de retardar o ponto de colheita, prolongar o período de armazenamento e aumentar o tamanho médio de frutos, permitindo assim maiores ganhos financeiros aos produtores.

Este trabalho objetivou avaliar os efeitos da aplicação pré-colheita de $\mathrm{GA}_{3}$ eAVG nas perdas, maturação na colheita e preservação da qualidade

${ }^{1}$ (Trabalho 128/2003). Recebido: 30/12/2003. Aceito para publicação: 09/03/2005.

2 Ph.D., Bolsista do CNPq. Centro de Ciências Agroveterinárias (CAV), Universidade do Estado de Santa Catarina (UDESC), Cx. Postal 281, CEP 88502-970, LagesSC. Fone (0**49) 221 2231. E-mail: amarante@cav.udesc.br.

${ }^{3}$ Estudante do Curso de Agronomia, CAV/UDESC. Bolsista de Iniciação Científica do CNPq. 
pós-colheita de pêssegos da cultivar Rubidoux.

\section{MATERIAL E MÉTODOS}

O experimento foi conduzido em um pomar comercial no município de Lages-SC, em 2001-2002. Foram utilizados pessegueiros com quatro anos de idade, da cultivar Rubidoux (cultivar tardia). O delineamento experimental foi em blocos inteiramente casualizados, com quatro repetições, consistindo de seis tratamentos, resultantes da combinação de duas doses de $\mathrm{GA}_{3}\left(0\right.$ e $100 \mathrm{mg} \mathrm{L}^{-1}$ ) e três doses de AVG (0; 75 e $150 \mathrm{mg}$ $\mathrm{L}^{-1}$ ). O GA 3 (com Silwet L-77 0,05\%) foi pulverizado em 16-12-2001, cerca de seis semanas antes do início da colheita comercial, correspondente ao período final de lignificação do endocarpo (formação do caroço), período em que frutos de caroço apresentam as melhores respostas à pulverização com GA, visando a retardar a maturação (Ju et al., 1999). O AVG (com Silwet L-77 0,05\%) foi aplicado em 10-01-2002, cerca de três semanas antes do início da colheita comercial.

Cada parcela foi composta de sete plantas, sendo as duas plantas das extremidades utilizadas como bordadura. Para avaliar a intensidade de queda pré-colheita, uma única planta de cada parcela teve todos os seus frutos contados durante o período de 17-01-02 a 9-02-02. As demais plantas tiveram todos os seus frutos colhidos nos dias 28 de janeiro e $1^{\circ}, 5$ e 9 de fevereiro de 2002 e avaliados quanto ao número, peso total, peso médio, percentagem de frutos rachados, percentagem de frutos com podridões e estádio de maturação. Foram colhidos todos os frutos de uma única planta por parcela em cada época de colheita.

Amostras de 15 frutos (com peso médio de 100-150 g) por parcela, de cada tratamento, livres de doenças e danos, em cada uma das épocas de colheita, foram avaliados quanto à maturação, um dia após a colheita. Os atributos de maturação avaliados foram a cor de fundo da casca, cor vermelha da casca, firmeza da polpa $(N)$, sólidos solúveis totais (SST; ${ }^{\circ}$ Brix) e acidez titulável. A cor de fundo da casca foi avaliada pelo emprego de tabela de cores numa escala de 1 (verde-escura) a 8 (verde-amarela). A firmeza da polpa foi quantificada com o uso de penetrômetro modelo Effegi, munido de ponteira com 7,9 mm de diâmetro. O teor de SST foi quantificado com o uso de um refratômetro de mão com compensação automática de temperatura (Abbe Atago). Em amostras compostas de suco extraído de cinco frutos, foram feitas as determinações de acidez (\% de ácido cítrico) através de titulometria de neutralização com $\mathrm{NaOH}(0,1 \mathrm{~N})$.

Amostras de 45 frutos (com peso médio de 100-150 g) por parcela, de cada tratamento, em cada uma das épocas de colheita, foram acondicionados em embalagens plásticas contendo sachês de pellets impregnados com permanganato de potássio (para absorção de etileno, sendo utilizado um sachê de $8 \mathrm{~g}$ para cada grupo de 45 frutos) e armazenados durante um mês em câmara fria convencional $\left(0-2^{\circ} \mathrm{C} / 90-95 \%\right.$ de umidade relativa). Um dia após remoção da câmara fria, amostras de 15 frutos foram avaliadas quanto à maturação, seguindo os mesmos atributos de maturação avaliados na colheita dos frutos, descritos anteriormente. Amostras de 30 frutos foram avaliadas visualmente quanto à incidência de doenças e de distúrbios fisiológicos (especialmente de escurecimento senescente da polpa) um dia após a remoção da câmara fria.

Os dados coletados foram analisados estatisticamente, usando o programa SAS (1990). As médias de tratamentos foram comparadas pelo teste $\operatorname{LSD}(\alpha=0,05)$. Visando a avaliar os efeitos de cada produto, foi efetuado contraste ortogonal linear entre tratamentos envolvendo doses de $\mathrm{GA}_{3}$ e análise de contrastes ortogonais polinomiais, para avaliar os efeitos de doses de AVG

\section{RESULTADOS E DISCUSSÃO}

A aplicação de GA ou AVG não foi efetiva em reduzir a queda pré-colheita de frutos (Figura 1). Somente na última data de avaliação (dia 09-02-2002), e quando aplicados em combinação (100 mg L-1 de GA e 75$150 \mathrm{mg} \mathrm{L}^{-1}$ de AVG), os produtos reduziram significativamente a queda précolheita de frutos (Figura 1).

O tratamento pré-colheita das plantas com GA $\mathrm{G}_{3}$ e AVG ocasionou retardo substancial na maturação de frutos, com maior retenção de cor de fundo da casca (cor verde), redução na cor vermelha da casca, melhor firmeza de polpa, redução no teor de SST e manutenção da acidez titulável,

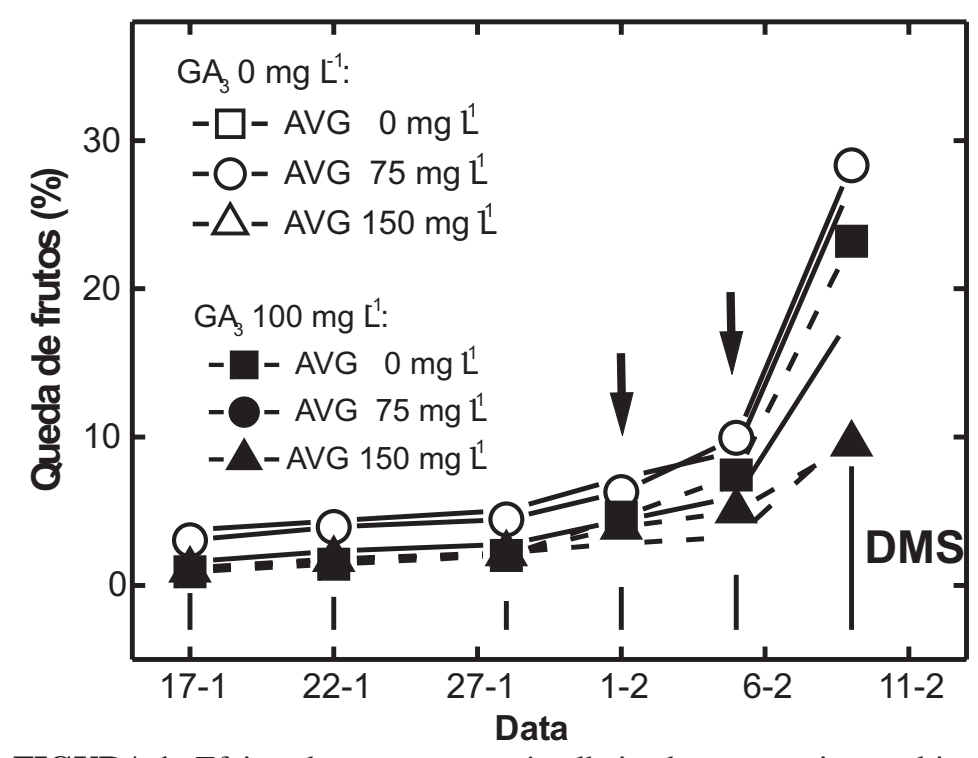

FIGURA 1 - Efeitos do tratamento pré-colheita de pessegueiros, cultivar

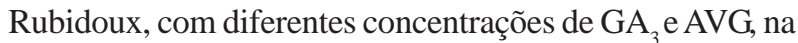
queda pré-colheita dos frutos. As setas indicam início e final do período de colheita comercial dos frutos. Diferenças mínimas significativas (DMS) entre tratamentos, indicadas no interior da figura, foram calculadas pelo teste LSD $(\alpha=0,05)$.

com o retardo na data de colheita dos frutos (Figura 2). De maneira geral, o maior retardo na maturação dos frutos foi observado pela aplicação isolada de $\mathrm{GA}_{3}\left(100 \mathrm{mg} \mathrm{L}^{-1}\right)$ do que pela aplicação isolada de AVG (75 e 150 $\left.\mathrm{mg} \mathrm{L}^{-1}\right)$. Estes resultados confirmam aqueles descritos por outros autores, mostrando maior retardo na maturação em pêssego pela aplicação précolheita de GA do que de AVG (Ju et al., 1999).

Não foi observado interação significativa entre época de colheita dos frutos e os tratamentos pré-colheita com AVG e GA 3 para todos os atributos de qualidade avaliados em pós-colheita. Desta forma, apenas os dados médios dos atributos avaliados, referentes às quatro épocas de colheita de cada tratamento, foram submetidos à análise estatística.

Os atributos de peso médio, de percentagem de frutos com rachaduras e podridões e de percentagem de perdas totais na colheita foram afetados pela aplicação pré-colheita de GA $\mathrm{G}_{3}$ e AVG (Figura 3). A análise de contraste ortogonal linear entre doses de GA mostrou que plantas tratadas com $100 \mathrm{mg} \mathrm{L}^{-1} \mathrm{de} \mathrm{GA}_{3}$ apresentaram, em relação a plantas não-tratadas com $\mathrm{GA}_{3}$, redução significativa $(\mathrm{P}<0,001)$ na percentagem de frutos podres (de $21,5 \%$ para $10,3 \%$ ), de frutos com rachaduras (de $14,3 \%$ para $9,0 \%$ ) e de perdas totais (podridões+rachaduras) (de 35,8\% para $19,2 \%$ ), e aumento significativo $(\mathrm{P}<0,001)$ no peso médio de frutos (de 113,6 g para $142,4 \mathrm{~g}$ ). No caso do AVG houve incremento linear significativo $(\mathrm{P}<0,001)$, com o aumento da dose de produto, na percentagem de frutos com rachaduras (de 8,5\% na dose de $0 \mathrm{mg} \mathrm{L}^{-1}$ para $13,6 \%$ na dose de 125 $\mathrm{mg} \mathrm{L}^{-1}$ ) e de perdas totais (podridões+rachaduras) (de 21,6\% na dose de 0 $\mathrm{mg} \mathrm{L}{ }^{-1}$ para $31,5 \%$ na dose de $125 \mathrm{mg} \mathrm{L}^{-1}$ ).

O incremento no peso médio de frutos em plantas pulverizadas com $\mathrm{GA}_{3}$ reflete o efeito promotor de elongação celular do produto, resultando em maior crescimento dos frutos (Taiz \& Zeiger, 1998). A redução no número de frutos rachados com a pulverização de $\mathrm{GA}_{3}$ pode ser o resultado do aumento na capacidade de expansão das células da casca, ocasionado pelo fitorregulador (Taiz \& Zeiger, 1998), reduzindo também a ocorrência de podridões, que ocorrem especialmente em frutos rachados. Em plantas não-tratadas com GA, o aumento nas doses de AVG ocasionou sensível aumento na percentagem de frutos rachados e com podridões, aumentando significativamente, assim, a percentagem de perdas totais na colheita (Figura 3). Nestes tratamentos, o aumento nas doses de AVG reduziu também sensivelmente o peso médio de fruto na colheita. $\mathrm{O}$ aumento nas doses de AVG reduziu, ainda, o tamanho de frutos na colheita em maçã (Amarante et al., 2002) e pêssego (Dekazos, 1981), e causou clorose foliar em melão (Shellie, 1999). Considerando que o AVG inibe outras enzimas que utilizam o piridoxal fosfato como co-fator enzimático, além da enzima ACC-sintetase (Taiz \& Zeiger, 1998), este produto, em dose elevada, pode 

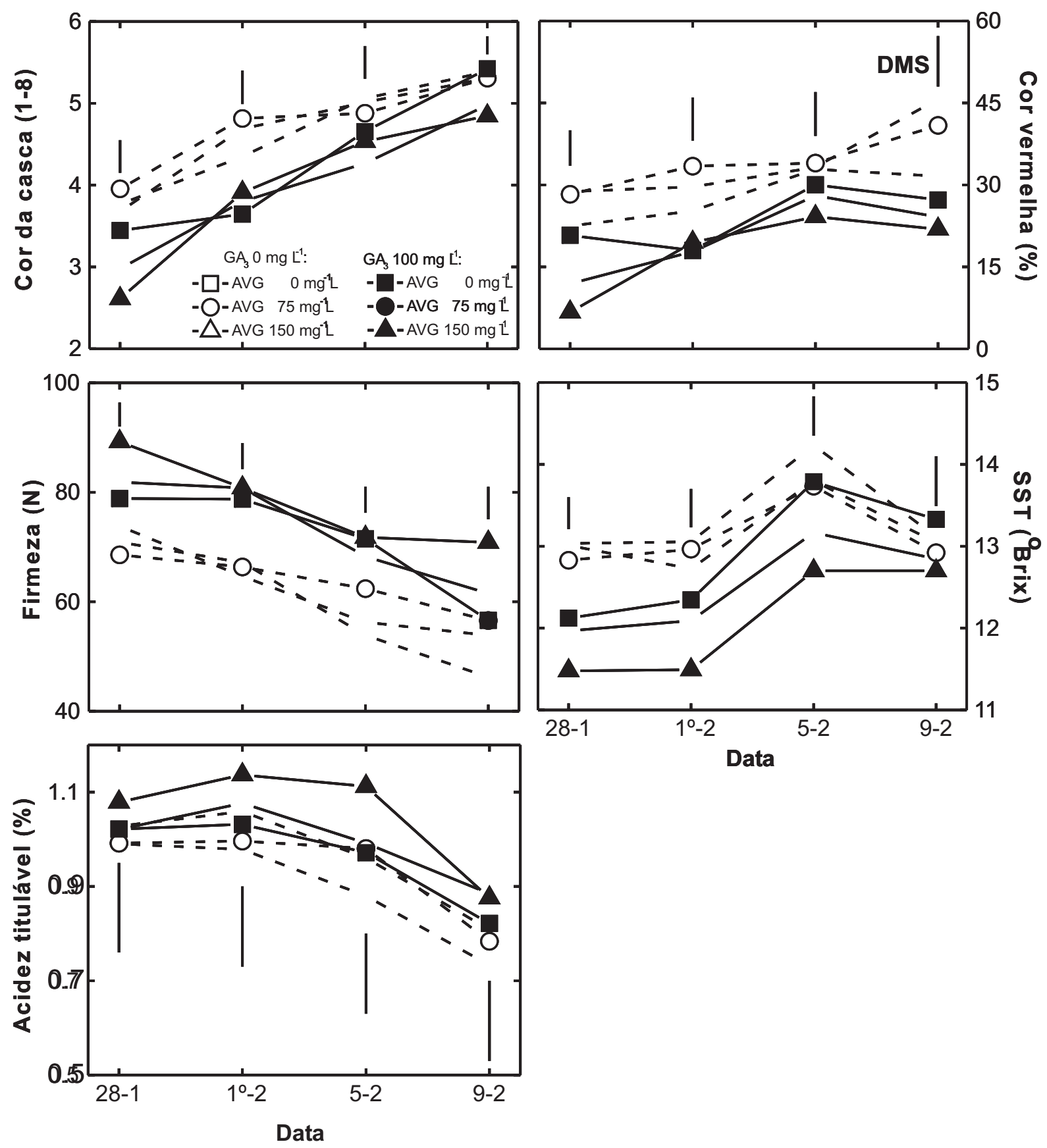

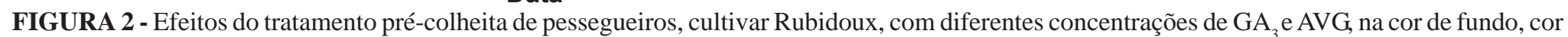
vermelha, firmeza de polpa, sólidos solúveis totais (SST) e acidez titulável de frutos colhidos em diferentes épocas. Diferenças mínimas significativas (DMS) entre tratamentos, indicadas no interior da figura, foram calculadas pelo teste $\operatorname{LSD}(\alpha=0,05)$.

inibir vários aspectos do metabolismo das plantas, inibindo assim o crescimento dos frutos, especialmente do tecido da casca. O tecido da casca não consegue crescer na mesma velocidade que o tecido da polpa (Faust, 1989), e, possivelmente, absorve e acumula maiores quantidades de AVG. Isto pode ter resultado em inibição da elongação celular e aumento na intensidade de rachadura da casca em pêssegos, com o aumento na dose de AVG, na ausência de tratamento com GA .

Frutos de plantas tratadas com $\mathrm{GA}_{3}$ e AVG, quando armazenados em câmara fria durante um mês, apresentaram melhor retenção de qualidade pós-colheita (Figura 4). O maior retardo no amadurecimento dos frutos foi obtido na combinação de tratamentos com GA, na dose de $100 \mathrm{mg} \mathrm{L}^{-1}$, mais AVG, na dose de $150 \mathrm{mg} \mathrm{L}^{-1}$. Isto mostra que, enquanto na colheita, $\mathrm{o}$ tratamento com $\mathrm{GA}_{3}$, na dose de $100 \mathrm{mg} \mathrm{L}^{-1}$, foi mais efetivo em promover retardo na maturação dos frutos, durante o armazenamento refrigerado, o tratamento adicional com AVG, na dose mais alta, resultou em melhores benefícios na preservação da qualidade dos frutos. Todavia, efeitos mais expressivos na preservação da qualidade foram observados pela aplicação isolada de $\mathrm{GA}_{3}\left(100 \mathrm{mg} \mathrm{L}^{-1}\right)$ do que pela aplicação isolada de AVG (75 e 150 $\mathrm{mg} \mathrm{L}^{-1}$ ). Frutos de plantas tratadas com $100 \mathrm{mg} \mathrm{L}^{-1} \mathrm{de} \mathrm{GA}_{3}$ apresentaram, em relação a frutos de plantas não-tratadas com $\mathrm{GA}_{3}$, maior retenção de cor de fundo da casca (cor verde) $(\mathrm{P}<0,001)$, maior firmeza de polpa $(\mathrm{P}<0,002)$ e maior acidez titulável $(\mathrm{P}<0,05)$. Com relação ao AVG, houve efeito linear positivo de dose do produto apenas na retenção de cor de fundo da casca $(\mathrm{P}<0,05)$ e de firmeza de polpa $(\mathrm{P}<0,05)$.

Frutos tratados com a combinação de AVG (75 e 150 $\mathrm{mg} \mathrm{L}^{-1}$ ) e GA (100 $\mathrm{mg} \mathrm{L}^{-1}$ ) apresentaram menor incidência de escurecimento senescente da polpa e de podridões após um mês de armazenamento refrigerado (Figura 5). Frutos tratados com $\mathrm{GA}_{3}$, na dose de $100 \mathrm{mg} \mathrm{L}^{-1}$, apresentaram os níveis mais baixos de escurecimento senescente da polpa, e, nos mesmos, o tratamento adicional com AVG não reduziu a percentagem de frutos com o distúrbio. Em frutos não-tratados com $\mathrm{GA}_{3}$, o aumento na dose de AVG reduziu substancialmente a incidência de escurecimento senescente da polpa. Isto parece indicar que o AVG, através da inibição da síntese do etileno, e, portanto, do processo de senescência, pode reduzir a incidência de escurecimento senescente da polpa durante o armazenamento refrigerado.

Estes resultados mostram que a aplicação pré-colheita de $\mathrm{GA}_{3} \mathrm{e}$ AVG apresenta potencial de uso comercial na cultura do pessegueiro para retardar a maturação e reduzir as perdas na colheita e durante o armazenamento refrigerado dos frutos. 

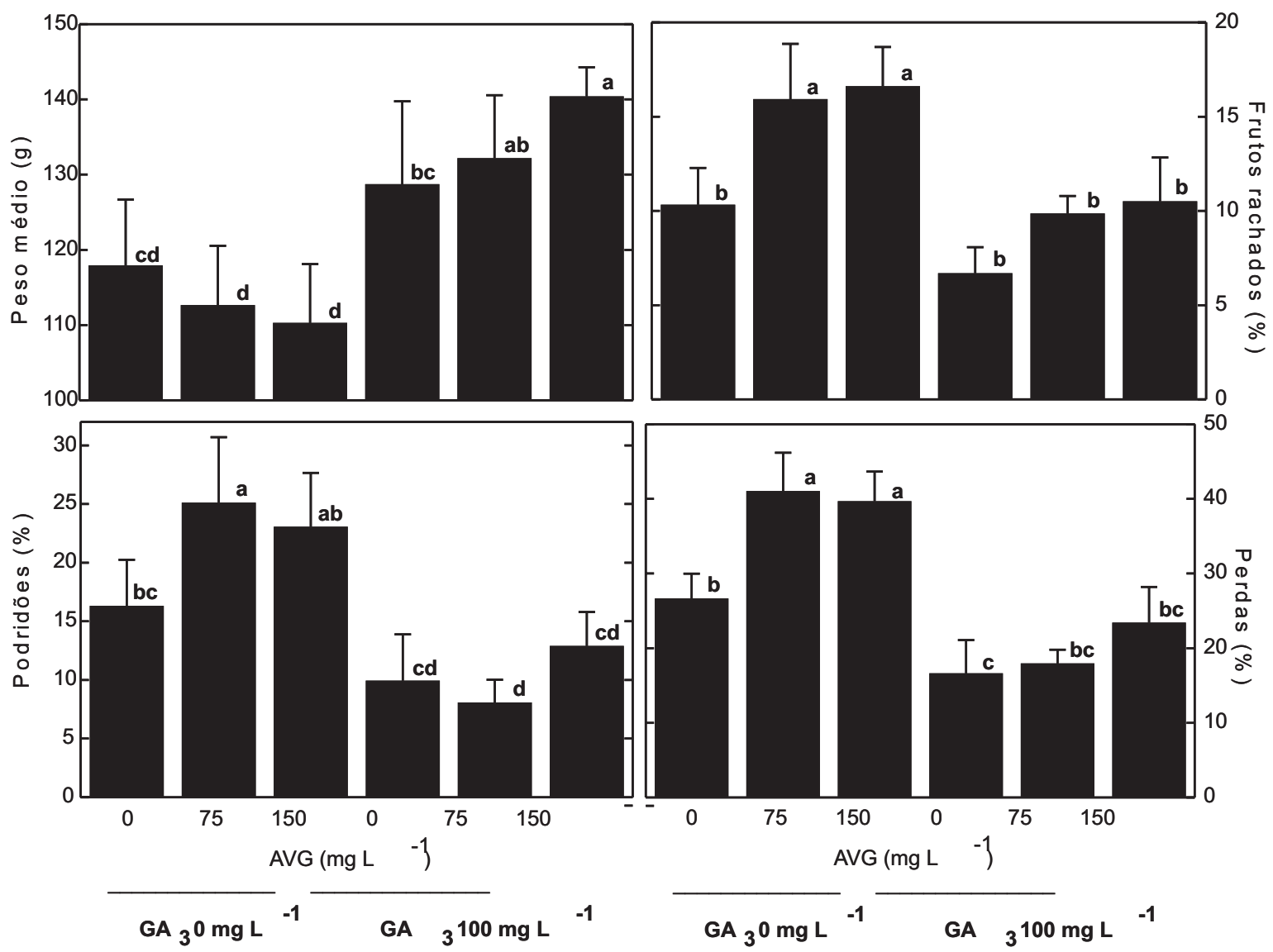

FIGURA 3 - Efeitos do tratamento pré-colheita de pessegueiros, cultivar Rubidoux, com diferentes concentrações de GA 3 e AVG, no peso médio de frutos, na percentagem de frutos com rachaduras e podridões, e na percentagem de perdas totais (resultante de rachaduras e podridões). Os valores representam a média de avaliações efetuadas em quatro épocas de colheita dos frutos (28-01, 1º-02, 5-02 e 9-02-2002). Médias seguidas pela mesma letra não diferem entre si, pelo teste $\operatorname{LSD}(\alpha=0,05)$. Barras verticais na parte superior das colunas indicam o desviopadrão da média.
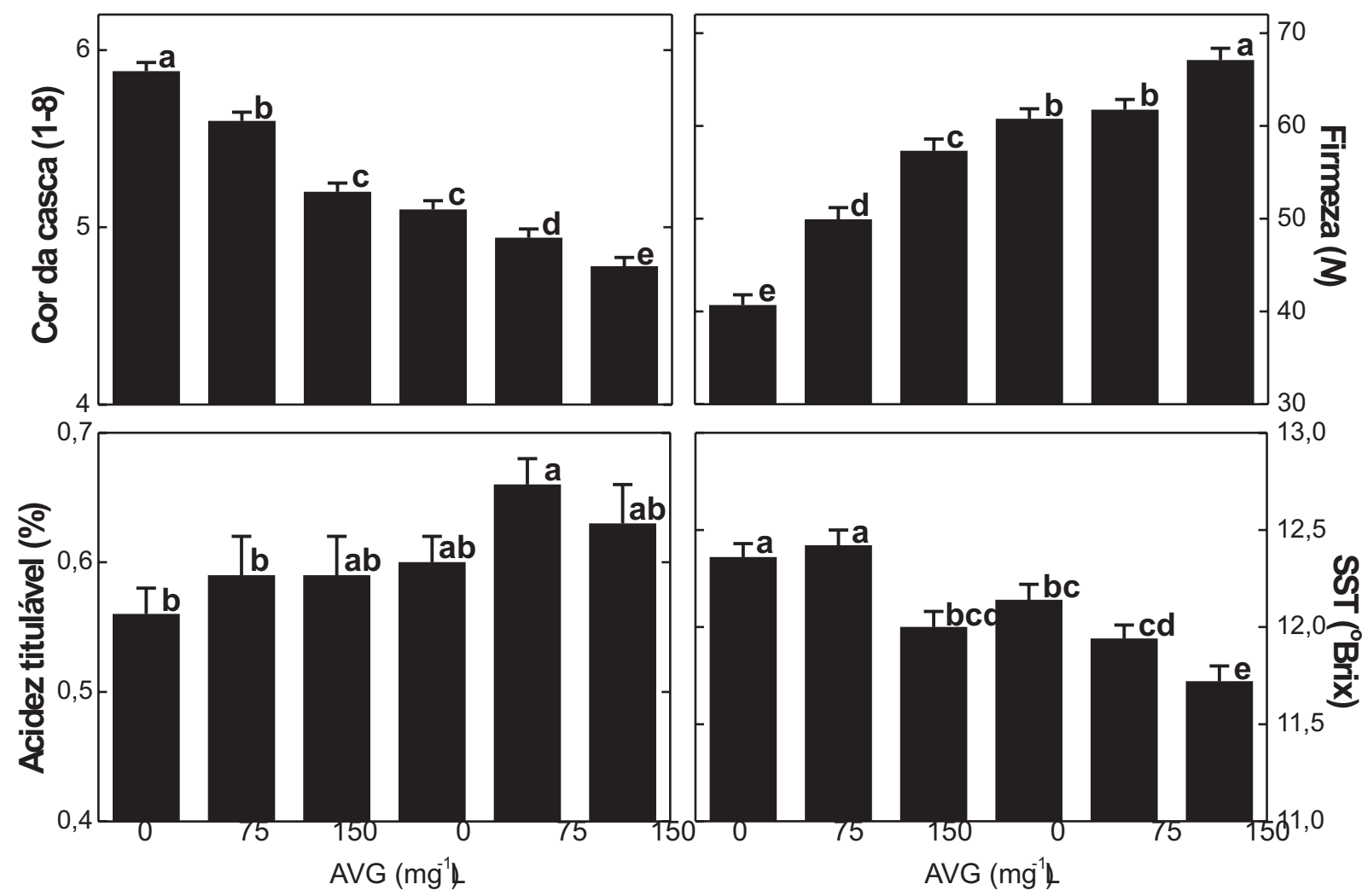

GA 0 mg L

\section{GA100 mg L}

GA 0 mg L

\section{GA100 mg li}

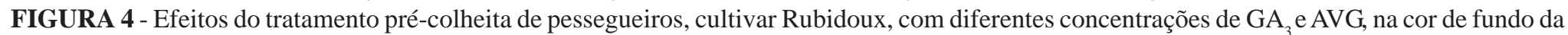
casca, firmeza de polpa, sólidos solúveis totais (SST) e acidez titulável dos frutos após armazenamento refrigerado. Os valores representam a média de frutos colhidos em quatro épocas (28-01, $1^{\circ}-02$, 5-02 e 9-02-2002) e armazenados durante um mês a $0-2^{\circ} \mathrm{C} / 90-95 \%$ UR mais 24 $\mathrm{h}$ a $20^{\circ} \mathrm{C} / 60-75 \% \mathrm{UR}$. Médias seguidas pela mesma letra não diferem entre si, pelo teste LSD $(\alpha=0,05)$. Barras verticais na parte superior das colunas indicam o desvio-padrão da média. 

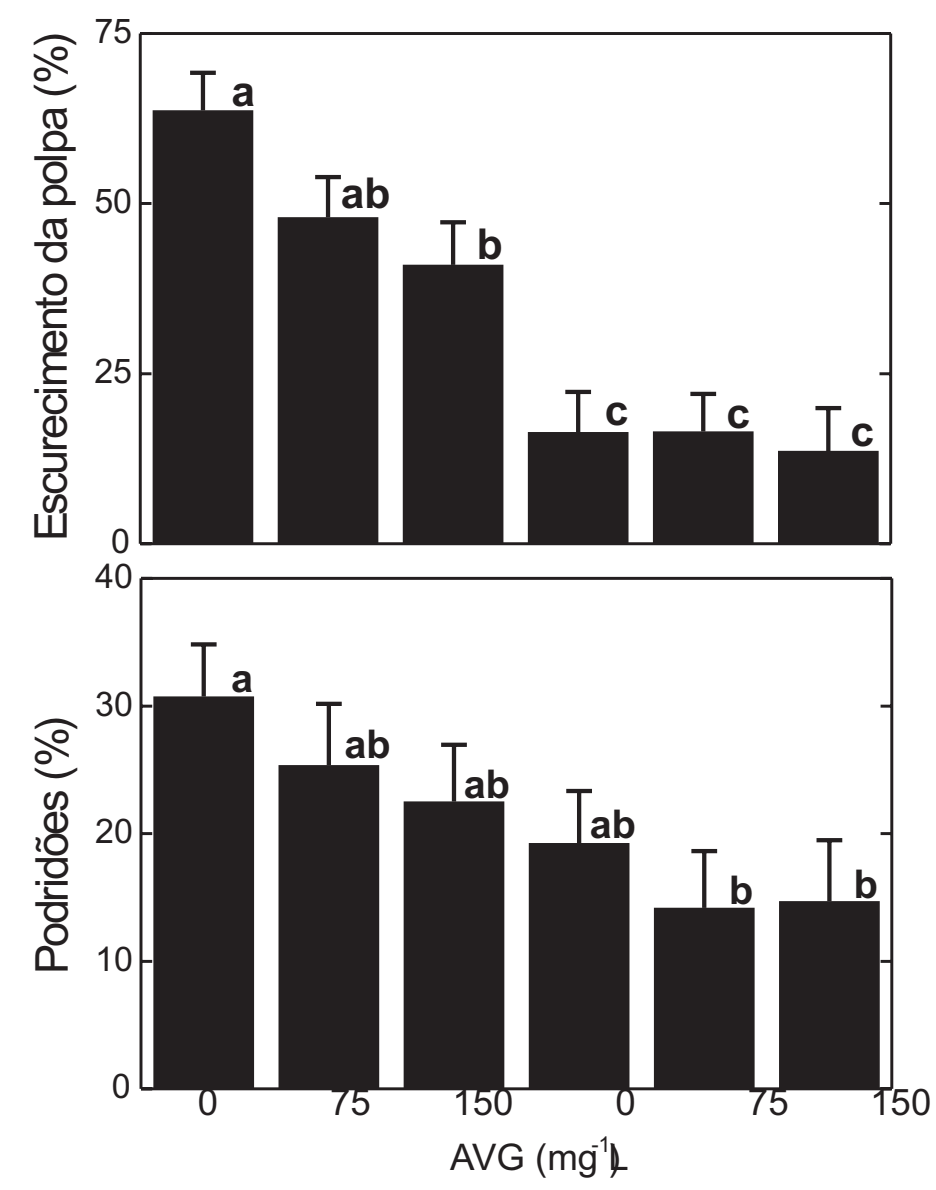

\section{GA $0 \mathrm{mg} \mathrm{L}^{1} \quad$ GA100 $\mathrm{mg} \mathrm{L}^{1}$}

FIGURA 5 - Efeitos do tratamento pré-colheita de pessegueiros, cultivar Rubidoux, com diferentes concentrações de GA e AVG, na incidência de escurecimento senescente de polpa e de podridões nos frutos após armazenamento refrigerado. Os valores representam a média de frutos colhidos em quatro épocas (28-01, $1^{\circ}-02$, 5-02 e 9-02-2002) e armazenados durante um mês a $0-2^{\circ} \mathrm{C} / 90-95 \%$ UR, mais $24 \mathrm{~h}$ a $20^{\circ} \mathrm{C} / 60$ 75\% UR. Médias seguidas pela mesma letra não diferem entre si, pelo teste $\operatorname{LSD}(\alpha=0,05)$. Barras verticais na parte superior das colunas indicam o desvio-padrão da média.

\section{CONCLUSÕES}

1. A pulverização pré-colheita com GA $\mathrm{A}_{3}$ AVG, especialmente em combinação, retardou a maturação e reduziu a incidência de escurecimento senescente da polpa em pêssegos.

2. Efeitos mais expressivos no retardo da maturação e na redução da incidência de escurecimento senescente da polpa foram mais observados pela aplicação de $\mathrm{GA}_{3}$ do que pela aplicação de AVG

3. $\mathrm{O} \mathrm{GA}_{3}$ ocasionou aumento no peso médio de frutos, menor incidência de frutos rachados e podres, e, portanto, menores percentagens de perdas totais de frutos na colheita comercial.

4. Em plantas não-tratadas com $\mathrm{GA}_{3}$, o incremento nas doses de AVG ocasionou aumento significativo na percentagem de frutos rachados.

\section{REFERÊNCIAS}

AMARANTE, C. V. T.; SIMIONI, A.; MEGGUER, C. A.; BLUM, L. E. B. Effect of aminoethoxyvinilglycine (AVG) on preharvest fruit drop and maturity of apples. Revista Brasileira de Fruticultura, Jaboticabal, v. 24, n. 3, p. 661-664, 2002.

AUTIO, W.R.; BRAMLAGE, W.J. Effects of AVG on maturation, ripening, and storage of apples. Journal of the American Society for Horticultural Science, Alexandria, v. 107, n. 6, p. 1074-1077, 1982.

BYERS, R.E. Peach and nectarine fruit softening following aminoethoxyvinylglycine sprays and dips. HortScience, Alexandria, n. 32, v. 1, p. 86-88, 1997.

DEKAZOS, E.D. Effect of aminoethoxyvinylglycine on bloom delay, fruit maturity, and quality of 'Loring' and 'Rio Oso Gem' peaches. HortScience, Alexandria, v. 16, n. 4, p. 520-522, 1981.

FAUST, M. Physiology of temperate zone fruit trees. New York: John Wiley \& Sons, 1989. 338p.

JU, Z.; DUAN, Y.; JU, Z. Combinations of $\mathrm{GA}_{3}$ and AVG delay fruit maturation, increase fruit size and improve storage life of 'Feicheng' peaches. The Journal of Horticultural Science \& Biotechnology, Kent, v. 74, n. 5, p. 579-583, 1999.

MARTÍNEZ-ROMERO, D.; VALERO, D.; SERRANO, M.; BURLÓ, F.; CARBONELL, A.; BURGOS, L.; RIQUELME, F. Exogenous polyamines and gibberellic acid effects on peach (Prunus persica L.) storability improvement. Journal of Food Science, Chicago, v.65, n.2, p. 288-294, 2000.

MATOS, C.S. Perspectivas à produção de pêssego tardio no Planalto Serrano: aspectos técnicos da cultura. Jornal da Fruta, Lages, n.92 ,p.10, 2000.

SHELLIE, K.C. Muskmelon (Cucumis melo L.) fruit ripening and postharvest quality after a preharvest spray of aminoethoxyvinylglycine. Postharvest Biology and Technology, Amsterdam, v. 17, n. 1, p. 55-62, 1999.

TAIZ, L.; ZEIGER, E. Plant physiology. $2^{\text {nd }}$ ed. Sunderland: Sinauer Associates, 1998. $793 \mathrm{p}$.

WILLS, R.; McGLASSON, B.; GRAHAM, D.; JOYCE, D. Postharvest: an introduction to the physiology and handling of fruits, vegetables and ornamentals. $4^{\text {th }}$ ed. Sydney: University of New South Wales Press, 1998. 262 p. 